http://dx.doi.org/10.18232/alhe.1175

Artículos

\title{
Competitividad y Estado en el complejo automotriz argentino a partir del análisis de su protección arancelaria (1965-1983)
}

\section{Competitiveness and State in the Argentine automobile industry from the analysis of tariff protection (1965-1983)}

Damián A. Bil ${ }^{1, *}$ (D) 0000-0002-7054-2927

${ }^{1}$ Universidad de Buenos Aires, Buenos Aires, Argentina.

* Correspondencia: damianbil@conicet.gov.ar

Resumen. En este artículo se analiza el esquema de protección efectiva para los sectores de autopartes y terminales automotrices en la etapa de su consolidación y posterior crisis (1965-1983). Inicialmente se propuso estudiar la forma de intervención del Estado para sostener la acumulación, sin embargo, la investigación abrió líneas complementarias de estudio, a saber: la relación económica entre autopartistas y terminales y las condiciones que permitían sostener la producción local de piezas o la importación de las mismas. Así, se analiza un aspecto de la intervención estatal en la industria y se aportan elementos para evaluar la performance del sector en su competitividad y las relaciones dentro de la cadena de valor.

Palabras clave: protección; aranceles; sector automotriz; autopartes; industria.

Abstract. We analyze the effective tariff protection for auto parts and automotive sectors in the large stage of industry consolidation and subsequent crisis (1965-1983). Our original idea was to study the form of state intervention to sustain the capital accumulation. The research itself opened additional lines of study: the economic relationship between auto parts and international assembly firms, or conditions that allowed

CÓMO CITAR: Bil, D. A. (2021). Competitividad y Estado en el complejo automotriz argentino a partir del análisis de su protección arancelaria (1965-1983). América Latina en la Historia Económica, 28(2), 1-22. DOI: 10.18232/alhe.1175 
local production of components or encourage the importation thereof. With this approach, we analyze one aspect of state intervention in industry and provide elements to evaluate the performance of the automotive industry in terms of competitiveness and relationships within the value chain.

Key words: protection; tariffs; automotive sector; auto parts; industry.

JEL: N46; N76; N86; N96.

Recibido: 09 de febrero de 2020.

Aceptado: 14 de octubre de 2020.

Publicado: 31 de marzo de 2021.

Organismo patrocinador: Consejo Nacional de Investigaciones Científicas y Técnicas.

Agradecimiento: Agradezco a los colegas Eduardo Sartelli e Ianina Harari, quienes revisaron la versión preliminar del presente trabajo y sugirieron correcciones y ampliaciones. Un especial agradecimiento a todo el personal del Centro de Documentación e Información del Ministerio de Economía de la Nación Argentina, que me brindaron el espacio y su hospitalidad para el relevamiento de buena parte de las fuentes y documentos que formaron parte de esta investigación.

\section{INTRODUCCIÓN}

La mayoría de los abordajes sobre la industria argentina se enfocó en el periodo posterior a la “crisis del 30", denominado como "industrialización por sustitución de importaciones" (ISI). Varios autores profundizaron sobre la función del Estado, como promotor u obstáculo de la actividad (Castellani, 2009; Fajnzylber, 1983; Racanello, 2010; Rougier, 2012; Teitel y Thoumi, 1986) o bien como partícipe de la producción (Belini y Rougier, 2008; Rougier y Odisio, 2016). En este marco, se estudian algunas de las formas de intervención en una rama particular como lo es la automotriz, en las condiciones de acumulación de capital en Argentina. En el presente artículo se analizarán los efectos de la política arancelaria y de protección efectiva sobre esta actividad.

En las décadas de 1970 y 1980, varios autores analizaron la estructura arancelaria en América Latina para determinar el nivel de protección efectiva (Altimir, Santamaría y Sourrouille, 1967; Balassa, 1975; Berlinski, 1977, 1978; Berlinski y Schydlowsky, 1982; Dadone y Swoboda, 1979; Katz y Kosacoff, 1989; Pinazo, 2015; Nogués, 1982; Szychowski, 1988; Wainer, 1970). Para el caso argentino, se buscó determinar el nivel de protección para la industria en general, entendida como el exceso porcentual del valor agregado bajo la protección respecto del mismo considerado a precios internacionales (Berlinski, 1978). La mayoría coincide en que hubo un complejo entramado de herramientas: recargos, aranceles, permisos de cambio e importación, diferentes tipos de cambio, entre otros.

Autores como Katz y Kosacoff (1989) sugieren que esto fomentó la producción de bienes finales, limitando la de maquinarias y equipos, y que las tarifas funcionaron como herramienta recaudatoria, igual que en el periodo previo a la crisis de la década de 1930. No obstante, la mayoría sostiene que, a partir de la crisis de 1930, la estructura arancelaria tuvo un efecto de protección real. En este caso, la discusión se presenta en torno a los niveles y a la racionalidad del esquema. Varios autores señalan que esto fue caótico, lo que generó una sobreprotección (Berlinski, 1977; Dadone y Swoboda, 1979; Wainer, 1970).

Más allá de estos debates, una preocupación era entender la articulación entre Estado e industria mediante el estudio de la protección del complejo automotriz entre 1965 y 1983 . Es decir, saber qué papel desempeñó la estructura arancelaria, combinada con otras medidas, en la acumu- 
lación de capital en la rama. El curso de la presente investigación abrió líneas complementarias como la relación entre autopartistas y terminales y las condiciones que permitían sostener la producción o la importación.

En cuanto a la estructura de protección, se originó en la crisis de comienzos de la década de 1950, que se sumó a la dificultad para importar bienes en los años de 1930 y durante la segunda guerra mundial. Esto provocó un envejecimiento del parque y una demanda insatisfecha. En este contexto, se declaró de interés nacional la fabricación de vehículos y partes, por lo que se instauró un esquema arancelario para proteger su producción. Se configuró un sector que tomó dimensión en el mercado interno, a nivel de producción y de empleo (Baranson, 1971).

A partir de la estructura arancelaria, un elemento a estudiar es lo específico del vínculo entre capitales de distinta magnitud en una trama productiva. ${ }^{1}$ Durante el periodo que se estudia, la relación entre terminales y autopartistas fue conflictiva: si bien las primeras se beneficiaban de la protección para vender sus vehículos en el mercado interno, también solicitaban liberalizar la importación de piezas y componentes, protegida por el sistema arancelario (Bil, 2018; Bil, Harari y Sanz, 2018). Estas tensiones estuvieron presentes en las negociaciones de los regímenes y decretos que regularon la actividad. En este sentido, una de las hipótesis es que la estructura arancelaria respondía en parte a la dinámica de estas negociaciones (determinadas por las relaciones de fuerza en la trama, las necesidades políticas y económicas vinculadas a la disponibilidad de divisas y otros factores). Asimismo, la existencia de una demanda insatisfecha junto a la protección del mercado interno aseguró una elevada rentabilidad para los participantes, sobre todo en los primeros años de instalación (Bil y Bastida, 2014; Fitzsimons y Guevara, 2018; Sourrouille, 1980). Como se señala, las terminales se quejaban de la política arancelaria, vinculada a los preceptos del compre argentino, ya que limitaba la capacidad de importar piezas y conjuntos más económicos o de mejor calidad. Se entiende que las diferentes valuaciones de aranceles para vehículos y partes, más elevados para los primeros, sería una forma de compensar lo que las terminales resignan por la obligación de comprar componentes locales.

En cuanto a la metodología utilizada, se procederá de la siguiente forma: en primer lugar, se reconstruirán los niveles arancelarios de las partidas correspondientes a productos automotrices y partes de los mismos durante el periodo estudiado, a partir de la información disponible en la Guía Práctica del Exportador y del Importador. Para ello se releva información de aranceles de importación para 133 partidas de autopartes y 45 de la industria terminal. Pero existen otros factores en el costo de importación, como la valuación del tipo de cambio. Por eso, para calcular la protección efectiva, se suma y resta a las alícuotas correspondientes de aranceles el nivel de subvaluación y sobrevaluación de la moneda local. El nivel de sobrevaluación y subvaluación de la moneda fue tomado de las series elaboradas por Juan Carrera (2007, p. 32), basado en la metodología de paridad relativa del poder adquisitivo. ${ }^{2}$ Con eso, se pretende una aproximación a los niveles de protección

${ }^{1}$ Se aclara que el presente artículo no estudia el sector en términos de cadena global de valor (Gereffi, 1999), sino que se refiere a un complejo productivo que consta de diversos subsectores. En este caso, industria proveedora (autopartista) y terminal (automotrices propiamente dichas). Se realiza un aporte al conocimiento de la estructura industrial en término de un complejo en un país para construir un bien final, diferente a lo que se conoce en la bibliografía como cadena global de valor.

${ }^{2}$ En cuanto a la metodología de cálculo del tipo de cambio, Carrera (2007) señala que "la relación cambiaria de dos monedas nacionales se encuentra en el nivel de paridad cuando el tipo de cambio vigente refleja de manera inmediata, al interior de cada país, la capacidad unitaria de su moneda para representar valor en el mercado mundial. Si determinadas condiciones de un proceso nacional de acumulación toman forma en políticas de su estado que ubican a su moneda en una relación de cambio con la otra por encima (debajo) del nivel de paridad, la moneda en cuestión 
efectiva de estos productos en la etapa bajo estudio. De esta manera, se aportan elementos para el análisis de la intervención estatal en la industria en Argentina y su incidencia sobre la acumulación, además de evaluar la performance del complejo automotriz en relación con su competitividad internacional.

El presente artículo se ordena de la siguiente forma: en primer lugar, se exponen las características de la acumulación en Argentina y el fundamento de la protección arancelaria en el país. Posteriormente, con ese marco, se reconstruye la historia del complejo automotor en Argentina en el periodo estudiado. Por último, se analiza la estructura arancelaria en la industria automotriz argentina en el sentido previamente desarrollado. Para avanzar en la investigación, se basa en el relevamiento de las fuentes señaladas, complementado con documentación de cámaras empresariales, estadística oficial, legislación y aportes de otras fuentes

\section{LAS CARACTERÍSTICAS DE LA ACUMULACIÓN Y LA PROTECGIÓN ARANCELARIA}

Posterior a la segunda guerra mundial, se perfiló un sistema de protección con la cobertura de aranceles a casi toda la industria. Los motivos son variados, se aducen razones de régimen, como el arribo de un gobierno nacionalista y el impulso de políticas de intervención. Aunque hay elementos más estructurales. Hacia comienzos de la década de 1950, Argentina se encontró en un momento de escasez de divisas por la merma de exportaciones agropecuarias. ${ }^{3}$ Por esta razón restringió la posibilidad de importar vehículos, aunada a la crisis de 1930 y la segunda guerra mundial, lo que provocó una demanda insatisfecha. Por ello, el parque automotor envejeció al igual que el de otros bienes de capital que se importaban. Para 1946, la cantidad de vehículos por habitante se redujo al nivel de 1934, y a mediados de la década de 1950 la edad del parque era de 20 años (Parrellada, 1970). Ante eso, se protegió el mercado interno y se fomentó la producción. Los elementos utilizados fueron el control del tipo de cambio y permisos de importación, subsidios, exenciones impositivas y, desde la década de 1960, fomento a la exportación de bienes no tradicionales. ${ }^{4}$ Existieron además regímenes de protección sectoriales, como en el caso automotriz.

A mediados de siglo se incorporaron diferentes gravámenes y recargos para la industria que complejizaron la estructura. Hasta 1947, los derechos aduaneros eran específicos y ad-valorem. La base imponible la marcaba el valor fijado en la tarifa de avalúos. Ese año se derogaron los aforos

se encuentra sobrevaluada (subvaluada). [...] El método utilizado consiste en identificar un periodo base en el cual el tipo de cambio vigente no actúa como vehículo de la apropiación de riqueza social, o sea, en que la capacidad unitaria de la moneda argentina para representar valor es similar hacia dentro y hacia el mercado mundial, para la luego acompañar la evolución de dicha capacidad respecto de uno y otro ámbito. [...] Se refleja la evolución relativa de la capacidad unitaria de las monedas nacionales para representar valor al interior de su ámbito nacional [...] mediante los respectivos índices de precios al consumidor [...] Si en un país la productividad del trabajo sube más lentamente que en el otro, la emisión de papel moneda adicional requerido para mantener los precios internos nominales en un nivel constante en cada uno de ellos implica que el papel moneda del primero está perdiendo su capacidad unitaria para representar valor respecto del segundo [...] el primero se estaría sobrevaluando [...]. El procedimiento para ajustar este eventual desvío consiste en tratar a la mayor productividad del trabajo en el segundo país [...] como si fuera una mayor intensidad del trabajo [...] el registro agregado de la productividad en el sector industrial resulte más apropiado como base del cómputo" (pp. 31-34).

${ }^{3}$ Este fenómeno de crisis del balance de pagos, conocido bajo distintas denominaciones (estrangulamiento de divisas, restricción externa, etc.) comenzó a ser recurrente en la economía argentina desde al menos esa década. El tema fue trabajado por varios autores, véanse Fiszbein (2013) y Schteingart (2016).

${ }^{4}$ Para un análisis más detallado, véase Bil, Dachevsky y Kornblihtt (2010). 
(salvo los acordados en tratados internacionales) y se exigió que se cobrara con base en el valor real de las mercancías (valor CIF) del importador. En 1955 se implementó el sistema de tipo de cambio diferencial e impuesto al comercio exterior: retenciones para exportaciones y recargos aduaneros para importaciones. Se establecieron cuatro categorías para las mercancías importadas: libre; de 20 \%; de 40 \% y depósitos previos de 150 a 200 \% por 180 días, y los de 300 \% con depósitos previos de $500 \%$. En 1959 se creó una intermedia sujeta a recargos del $100 \%$, donde se pasaron productos sujetos, hasta entonces, a fuertes gravámenes. Se exceptuó al equipamiento agropecuario, forestal, máquinas para explotación petrolera y carbonífera, buques y aeronaves, importaciones del Estado e inversiones de capital extranjero aprobadas por el gobierno, además de bienes de capital cuando no se fabricarán en el país.

Entre 1964 y 1965 se derogó este sistema, quedando solo un gravamen por partida bajo el nombre de derecho de importación. Se establecía que las importaciones podían recargarse con impuestos destinados al fomento de sectores o emprendimientos públicos (siderurgia, minería, explotación forestal, marina mercante). De igual forma, el gobierno podía prohibir la importación de productos, ${ }^{5}$ o bien gravar algunos con aranceles prohibitivos. En agosto de 1962, la partida "automóviles cuyo peso y/o costo en fábrica [...] sean superiores en kilogramos a $1500 \mathrm{y} / \mathrm{o}$ U\$S 2 000” contaba con un arancel nominal del $1000 \%$ (Guía Práctica del Exportador y del Importador, 1962). El ejecutivo podía flexibilizar el ingreso mediante recargos menores, sobre todo cuando se hacía necesaria la importación.

Asimismo, existían tratamientos con reducción de aranceles según el país de origen para los firmantes de tratados, como el de la Asociación Latinoamericana de Libre Comercio o General Agreement on Tariffs and Trade, formas de reforzar la restricción mediante la modificación de aranceles por decreto o el sistema de autorizaciones para importar según las necesidades (Dadone y Swoboda, 1979). El gobierno militar que asume el poder tras el golpe de estado de marzo de 1976 estableció un plan de apertura que modificó en gran medida la estructura de las décadas previas. En 1978 se estableció un arancel nominal máximo, y a partir de eso una reducción trimestral de los mismos para permitir la adecuación de los diferentes sectores al nuevo marco (Rezk y Ruatta, 1980), para otros autores (Sourrouille, Kosacoff y Lucángeli, 1985) en realidad se aplicó un shock en la estructura de protección. Para estos últimos, el gobierno militar intentaría entonces

la erradicación inmediata, lisa y llana, de la "ineficiencia", cualquiera fuese el origen de la empresa, generando una nueva base productiva industrial ligada a un tipo de cambio real ajustado sustantivamente, en particular en cuanto al nivel arancelario medio prevaleciente. [...] desde fines de 1978, combinando un muy fuerte reajuste arancelario, una sostenida revaluación del peso y la consiguiente caída del tipo de cambio real (pp. 59-60).

Los mismos autores citados señalan que el plan económico del ministro Martínez de Hoz en este punto buscaba, al menos discursivamente, una mejora en la eficiencia productiva (p. 105). Se caracterizaba que los aranceles eran elevados y dispersos, por ello se aplicó a fines de 1976 una primera tanda de medidas para eliminar la redundancia. En 1978 se dio paso a una reforma general que intentó llevar el nivel promedio a $15 \%$ (p. 77). A comienzos de 1979 se oficializó un nuevo programa que ajustó el nivel nominal de protección y redujo la dispersión, sobre todo en el sector

${ }^{5}$ Por ejemplo, en enero de 1964 se prohibió la importación de mercancías incluidas en la partida Automóviles y coupés (Guía práctica del exportador y del importador, 1964). 
de bienes de capital. Pero en el sector automotriz las terminales pudieron mantener ciertos privilegios en la protección de vehículos y flexibilizar la importación de piezas (p. 109), reclamo de las principales automotrices desde hacía años. Kosacoff y Azpiazu (1989, p. 14) agregan que, a pesar de la baja en promedio de la protección nominal, en los primeros tres años del Proceso Militar las importaciones no aumentaron de forma masiva. Sin embargo, la profundización de la reforma con un proceso de sobrevaluación monetaria generó una apertura masiva de importaciones. Aun así, como se verá, para el caso automotriz esto es relativo: si bien entre 1980 y 1981 las importaciones de componentes y vehículos se multiplicaron, fueron limitadas por la crisis económica y de balance de pagos.

Así, se coincide con los señalamientos de otros autores (Rezk y Barra Ruatta, 1980, Carrera, 2007), la estructura estaba cruzada por otras variables, como el tipo de cambio, pues un dólar barato facilitaría las importaciones de bienes, caso contrario dificultaría las compras al extranjero y actuaría como protección para la producción local. A este factor se suma el entramado de aranceles (y también permisos o prohibiciones de importar mercancías producidas localmente) para dar un nivel de protección efectiva. De esta forma, se propone analizar en el presente estudio cómo operó todo el esquema reseñado en este caso particular.

\section{EL COMPLEJO AUTOMOTRIZ DURANTE LA SEGUNDA MITAD DEL SIGLO XX}

Durante las décadas previas a la segunda guerra mundial, la difusión de la producción en serie en Estados Unidos posibilitó el ensamblado en los mercados consumidores. La economía de escala era mayor en la producción de partes que en el armado, lo que fomentó que se instalaran plantas en otras plazas. En América Latina, Ford instaló las primeas en Argentina y Brasil durante la década de 1910 (Wilkins y Hill, 2011) con base en semi knocked down (SKD) ${ }^{6}$ y completly knock down (CKD), ${ }^{7}$ conjuntos prearmados para ensamblar en el destino. A partir de la década de 1950, la producción mundial se expandió, con la incorporación de los primeros sistemas de automatización en producción de piezas. Estos cambios, y la profundización de la competencia internacional, permiten explicar la consolidación automotriz en América Latina: los japoneses y los europeos instalaron fábricas con estas nuevas tecnologías, mientras que el capital estadunidense contaba aún con inversiones sin amortizar. Por ello, la inversión de estas firmas en América Latina se dio como vía para acelerar el cambio técnico en los países clásicos, por exportación de instalaciones obsoletas en aquellos mercados hacia esta región, donde lograron valorizarse y permitieron la renovación tecnológica en Estados Unidos (Fitzsimons, 2013; Grinberg, 2011; Harari, 2011; Nofal, 1989, p. 90).

Para Argentina, este fenómeno coincide con factores internos: por un lado, la demanda de piezas y repuestos para mantener operativo el parque, que ante las dificultades para importar desde la década de 1930 generó el crecimiento de talleres y pequeñas manufacturas para el mercado de reposición, esta fue la base para la expansión de la industria de proveedores ocupada por fracciones de la burguesía local (Nofal, 1989, p. 13); por otro lado, en 1951 la automotriz fue incorporada al régimen de promoción, en 1953 la Ley de Inversiones Extranjeras posibilitó que se instalaran multinacionales, de esta forma llegaron Industrias Kaiser Argentina y Ford, Citröen, y otros, y se instalaron nacionales como Siam, Industria Automotriz Santa Fe S. A., Autoar, y otras que tuvieron una escasa trayectoria como Isard o Marimón, entre otras.

${ }^{6}$ Kit con el cuerpo del auto, con algún revestimiento o ya pintado, para entonces incorporar el motor en el país receptor.

${ }^{7}$ Kit de montaje. 
En marzo de 1959 se firmó el decreto 3693 (Régimen de Promoción de la Industria Automotriz), que elevaba aranceles a terminados o directamente prohibía la importación de vehículos (aunque mantuviera aranceles a título indicativo); además, las plantas que utilizaran partes nacionales recibirían ventajas cambiarias y arancelarias, y se les garantizaría la provisión de divisas para equiparse. Entre 1959 y 1960 se aprobó la instalación de 23 firmas, aunque para 1964 once ya habían abandonado la producción. ${ }^{8}$

A pesar de que el objetivo fue reducir el drenaje de divisas y montar una producción sin déficit, la balanza automotriz continuó siendo deficitaria. Nofal (1989) calcula que de 1959 a 1964 en promedio se importaban partes por 738 dólares por vehículo. El costo de importación de partes doblaba la inversión de los fabricantes extranjeros (Nofal, 1989, pp. 31, 144). Los fabricantes hicieron un uso provechoso del sistema permisivo de cuotas y tasas y obtuvieron abundantes beneficios (Fitzsimons, 2013). Terminales que se integraban para producir localmente, como Industrias Kaiser Argentina, se quejaban de la falta de mecanismos de control y de la ventaja desleal que tenían otras compañías (Industrias Kaiser Argentina, 1963, pp. 65-66), por ello, se modificaron los regímenes. Bajo la presidencia de Illia (1963-1966) se aumentó la obligatoriedad de integración nacional. Se pretendía llegar a 96 \% de composición local en 1968 (Sourrouille, 1980, p. 57). El promedio de importación por unidad cayó a 294 dólares según Nofal (1989), aunque Baranson (1971) calculaba 371. Algunas firmas ampliaron instalaciones para producir piezas, y para fines de la década de 1960 todas tenían líneas de estampado y ensamble de carrocerías, mecanizado y montaje, pintura, tapizado y ensamble. Otras instalaron, además, mecanizado y montaje de conjuntos (Parrellada, 1970, p. 6), aunque se prefería en lo posible tercerizar la producción de autopiezas a proveedores, dada la baja escala de la producción local.

En este contexto, se desarrolló el autopartismo. Para mediados de la década de 1960, existían firmas que estampaban chapa, ensambles de carrocería, pintura, forjado y mecanizado, de fabricación de conjuntos, entre otras. En ese sentido, Cimillo, Lifschitz, Gastiazoro, Ciafardini y Turkieh (1973) señalan que la transferencia de plusvalor de los pequeños capitales autopartistas a las multinacionales limitó la acumulación en el sector. No obstante, en términos empíricos se observa que el sector se modernizó con el crecimiento de compañías locales y también con la radicación de capital extranjero, con inversiones en firmas nacionales o la cesión de licencias de sus productos, asimismo se dio un proceso de concentración (Bil, 2017). El crecimiento fue segmentado, ya que las grandes firmas se dedicaron a proveer a las terminales, mientras que las pequeñas quedaron relegadas al mercado de reposición. Para 1973, los pequeños establecimientos generaban solo $8 \%$ de la producción (Barbero y Motta, 2007, p. 202).

La industria autopartista se expandió gracias al régimen de promoción que la obligaba a integrarse nacionalmente, mientras que las terminales que no cumplían pagaban un recargo. En 1964, la integración exigida era $90 \%$ para autos y $80 \%$ para camiones, así se buscaba reducir la importación y la necesidad de divisas. Estas normativas generaron disputas, particularmente hacia comienzos de la década de 1970. Tal es así que en 1971 se produjo un conflicto por la renovación del régimen legal (Harari, 2010). Los representantes de las terminales, unidos en la Asociación de Fabricantes de Automotores (en adelante ADEfA), y los autopartistas nucleados en la Cámara Industrial de Fabricantes de Autopartes de la República Argentina (en adelante CIFARA) reconocían que el complejo tenía altos costos, aunque eran conscientes de problemas generales como los

${ }^{8}$ Un tratamiento en detalle de las normativas y evolución de la automotriz puede verse en Feder (2019), Fitzsimons (2013, 2016), Harari (2011) y Sourrouille (1980). 
costos de materias primas o la baja productividad por el mercado acotado, ambas partes se culpaban mutuamente. Las terminales acusaban a las autopartistas de ser ineficaces por carencia de equipamiento, lo que motivó inversiones adicionales en las armadoras para estampado, forjado y mecanizado.

Salvo excepciones, los establecimientos fabricantes de partes no cuentan con todos los equipos específicos de las tecnologías que abastecen. Lo corriente es encontrar maquinaria convencional que, aunque sea relativamente nueva en cuanto a años de uso, presenta diseños que en general corresponden a proyectos antiguos [...] el empleo de técnicas avanzadas, [...] demandaría inversiones que sólo podrían ser absorbidas para niveles de producción de las terminales muy superiores. [...] Las excesivas inversiones [...] que obedecen a las razones antes citadas, configuran el panorama actual de una industria terminal que presenta un equipamiento excesivo frente a las necesidades del mercado, lo que redunda en una capacidad ociosa [...]. Esa capacidad ociosa se manifiesta a través de la comparación entre la capacidad máxima de producción posible de las empresas asociadas a ADEFA, que es de aproximadamente 242000 unidades y de la producción efectivamente realizada en el año 1967 de 149000 unidades, o sea un $38.39 \%$ de capacidad productiva no saturada. Los elevados porcentajes de nacionalización de los países en vías de desarrollo han colocado a las empresas internacionales ante algunas alternativas de difícil resolución. Uno de ellos es que [...] para no perder mercados se han visto en la necesidad de instalar en todo el mundo fábricas dimensionadas para pequeños volúmenes [...] mientras que por el otro, la tecnología y las condiciones para competir internacionalmente las impulsan a una concertación de esfuerzos [...] los países en vías de desarrollo han insistido en la creación de una industria nacional del automotor que, debido a los pequeños volúmenes de producción, presenta características de trabajo intensivo (ADEFA, 1969, p. 34).

La cámara de industriales automotrices manifestó no estar contra la integración nacional, sino contra el elevado porcentaje. Pedían que este no superara 40 u 80 por ciento.

Este 40 \% de nacionalización es el límite máximo económico para los tramos inferiores dentro [...] de los países en vías de desarrollo. Está compuesto por el montaje y por la fabricación de partes que se obtienen mediante procesos tecnológicamente sencillos o bien que poseen en el mercado de reposición una importante demanda creciente, lo que les permite alcanzar mayores volúmenes de producción. Por encima del $40 \%$ y hasta un $80 \%$ ya se entra en la fabricación de los equipos eléctricos [...] y de los componentes y partes [...] del motor, caja de cambios y diferencial, con las economías de escala que es fácil suponer [...]. Este valor del 75/80\% aparecería como el límite máximo teórico de nacionalización que, a falta de otras razones [...], tendría que alcanzar la industria automotriz en los países en vías de desarrollo. Superando el $80 \%$ se requieren fuertes inversiones en la matricería [...], tropezándose además con los inconvenientes propios del proceso de estampado en pequeña serie [...] (ADEFA, 1969, p. 41).

Mientras, las autopartistas negaban su responsabilidad en la elevación de los precios de vehículos. Señalaban la disminución de la productividad de las terminales como elemento primordial, y proponía el resguardo del autopartismo como de interés nacional (Broner y Larriqueta, 1969; 
CIFARA, 1970, p. 27). Julio Broner, director de la firma de embrague Wobron, y que llegó a ser presidente de la Confederación General Económica (CGE) en 1974, destacaba en un plan de trabajo de CIFARA:

A pesar de los más de 10 años transcurridos desde el dictado del régimen del decreto $3693 / 59$, se prevé mantener por tiempo indeterminado una zona vedada para la industria nacional de autopiezas, constituida por los márgenes de importación del 5 y 13 por ciento. [...] ello conspira contra posibilidad de que la industria nacional de autopiezas encare la fabricación local de algunos elementos que actualmente se importan, ya que además los derechos preferenciales operan directamente a favor del principal consumidor, o sea la industria terminal. [...] El gasto de divisas que ocasionan los márgenes admitidos de importación son considerables y durante 1969 se han incrementado notablemente. [...] Teniendo en cuenta estos antecedentes entendemos imprescindible fijarse una política para el remplazo de estas importaciones por producción local, y su compensación con exportaciones del sector (Taraciuk, 2015, p. 77).

Además de esto, el tamaño del mercado afectaba a la industria autopartista, cuya escala resultaba chica y limitaba la tecnología empleada. La calidad de los productos solía ser deficiente para el caso de material eléctrico, forjado, fundiciones, cromados, plásticos y vinílicos, además de las carrocerías por falta de chapas acordes (Baranson, 1971, p. 60). La baja productividad implicaba que los precios de sus productos se encontraran por encima del costo de las importadas. En ese contexto, las terminales pugnaron para reducir el cupo de integración local y poder importar piezas. Pero la posición que primó fue la de las autopartes: el Régimen de Reconversión aceptó ciertas reivindicaciones de las terminales, como la prohibición de importación de vehículos hasta 1980, pero en su conjunto benefició a las autopartistas. Estas obtuvieron el sistema de listas positivas, donde el recargo para importación se establecía según la disponibilidad de cada pieza en el mercado local; además se fijaron topes de entre 5 y $10 \%$ de importación para autos y camiones (una leve reducción de la situación previa) y un reintegro de 50 \% para las que exportaran. El golpe de 1976 cambió la relación de fuerzas entre ambas fracciones de la burguesía automotriz. La redefinición de los términos del régimen automotriz hacia fines de la década de 1970 generó el beneplácito de las terminales. Los industriales denunciaban que la elevada integración aumentaba los costos, deprimía las ventas e imposibilitaba lograr economías de escala (Mercado, 1982). Ante el nuevo régimen el presidente de Ford Argentina, Courard, señalaba

el régimen de reconversión tiene una inspiración positiva por cuanto busca, una mayor eficiencia dentro del sector y permite, por ejemplo, un mayor contenido de partes importadas con incorporación de tecnología de último nivel que en algunos casos sería innecesario y muy costoso desarrollar localmente, dado los volúmenes con que opera la industria local (Mercado, 1980, p. 26).

Carlos Mandry, de Volkswagen y presidente de la ADEFA, agregaba que

en todo el mundo la industria automotriz baja los costos y mejora la economía de escala mediante un activo intercambio de piezas, que actualmente en la Argentina está muy restringido por las regulaciones oficiales [...]. Si con esas grandes inversiones se producen series muy cortas de productos, entonces los costos son altísimos por la 
incidencia [...] de esa gran inversión por unidades. [...] esas grandes inversiones pueden ser canalizadas hacia series de producciones más grandes destinadas al consumo interno y a la exportación, e importar los productos de los cuales se necesita poca cantidad. De esta forma no se necesitaría hacer grandes inversiones en todos los rubros. [...] Si la integración nacional es muy alta, los costos son altos, los precios son altos, se vende poco y no se logra la economía de escala (Mercado, 1982).

Además de la cuestión de los aranceles y la alícuota de integración nacional, existían otros problemas. Uno de ellos era el proceso productivo. Existía un déficit en matricería, donde por escasez de las necesarias se debían adaptar, aumentando el tiempo de producción y su costo. El informe de Baranson (1971) para el Banco Mundial aseguraba que "en las prensas de estampación de las carrocerías hay que cambiar varias veces las pesadas matrices para ir fabricando en pequeñas series los 20 o 30 paneles de chapa que lleva cada modelo de coche o camión” (p. 60). Esto significaba un encarecimiento de los precios. Sourrouille (1980, p. 63) mencionaba que el consumidor argentino pagaba 2.2 veces el precio de un automóvil equivalente a los precios internacionales debido a la baja economía de escala, problemas de gerenciamiento, y déficits en insumos. Por su parte la ADEFA, la cámara que unía a las terminales, tenía una evaluación similar (ADEFA, 1969, p. 51).

Otros estudios del periodo dieron cuenta de este diferencial de costos. Se señalaban los déficits de la industria de base, con mayores costos y dificultades de aprovisionamiento, extendían esos déficits al resto de los eslabones. Las piezas producidas en el país eran costosas a nivel internacional. Las del sector metalúrgico eran 1.96 veces más caras que el precio promedio internacional, los conjuntos eléctricos 2.2, los neumáticos 2.24 y las partes de caucho 1.64. Estas diferencias repercutían en el costo final (véase gráfica 1 ).

Además, la escala era reducida, ya que se calculaba que el volumen mínimo de producción para poner en funcionamiento una línea automotriz, con ensamblado y producción de motores como en el caso argentino, era de 250000 unidades anuales con la tecnología más avanzada, siendo 600000 el óptimo en Estados Unidos (Remes, 1974). La escala debía aumentar si se incorporaba la producción de piezas, debido a que estos procesos se encontraban más mecanizados que el ensamble. En aquellas secciones donde la inversión en maquinaria era mayor, también lo era la escala necesaria: 


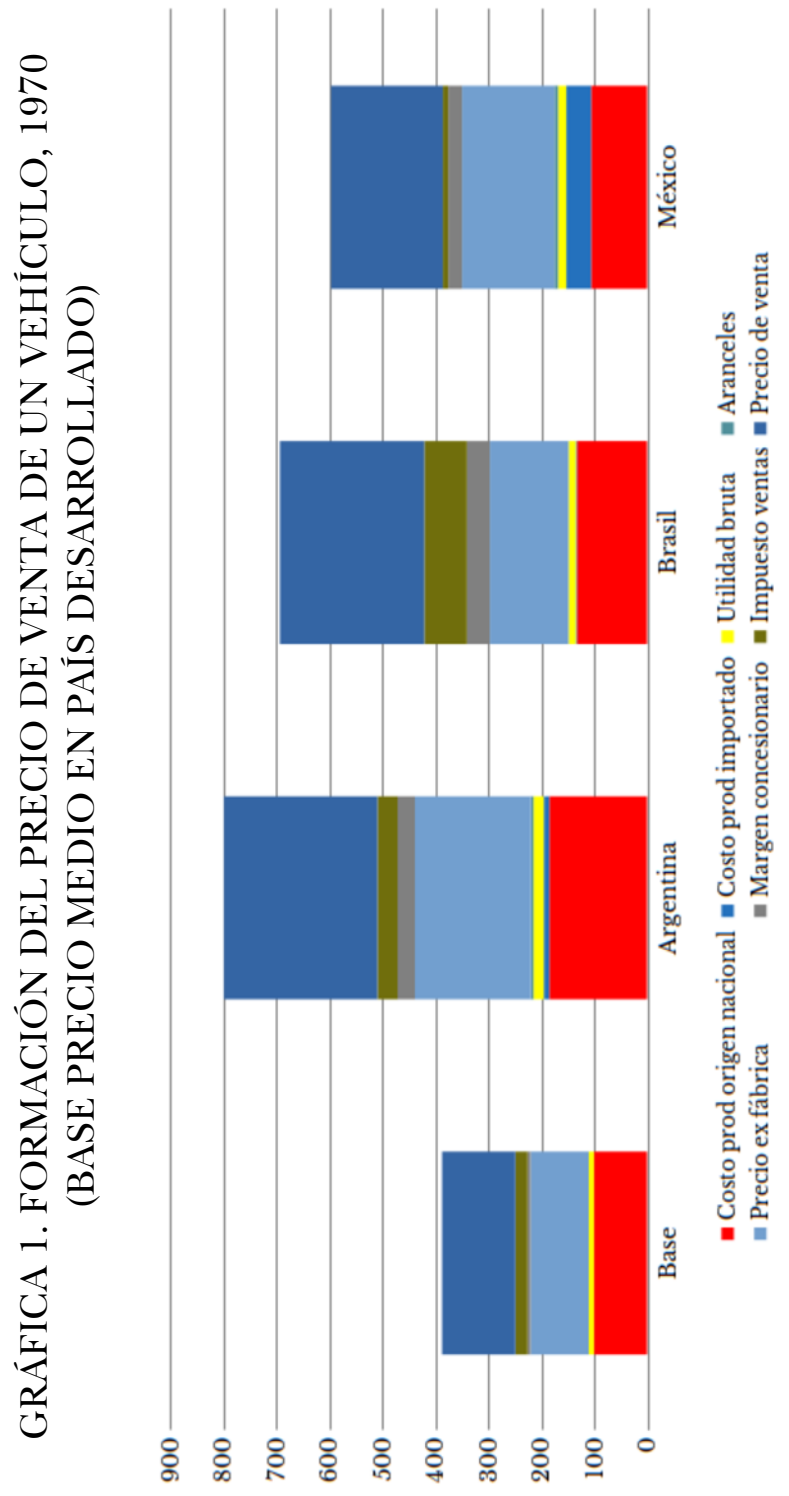

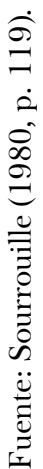


Para justificarlas y amortizarlas se necesita una conveniente producción, que en las grandes empresas extranjeras llega hasta a varios miles de unidades por día. Reduciendo la producción a las medidas de nuestro mercado, es decir un total de unos centenares de unidades por día, disminuyen las inversiones, pero como consecuencia final aumentan los costos unitarios del producto. Lo dicho para la industria terminal va también para la industria auxiliar, empeorando el panorama la falta, casi general, de modernos equipos de producción; lo que justifica la señalada necesidad de reequipamiento de este sector industrial. En un análisis detallado de esta situación, hemos encontrado en este caso particular más o menos los mismos guarismos de costos nacionales que se han indicado para automotores completos [...] La conciencia industrial, especialmente en las pequeñas industrias auxiliares, todavía insuficiente para el entendimiento de lo riguroso que es para la industria terminal el término de entrega, la falta del cual en un elemento de ínfimo valor, impide la entrega de una costosa unidad automotriz, inmovilizando capitales valiosos. Por otro lado, obliga a mantener stock de elementos y materiales con una inmovilización de capitales, cuyo costo se vuelca directamente sobre el producto (Rossi, 1960, p. 14).

El límite del mercado interno provocaba que las terminales operaran con capacidad ociosa (alrededor de $40 \%$ ), por lo que traer equipos de mayor capacidad hubiera sido antieconómico. En 1965, mientras en Estados Unidos se producían 11112000 de vehículos, en Argentina se fabricaron solo 196 800. En 1980, Argentina vendió 346716 unidades; en Japón casi 5000000 y en Estados Unidos, en plena crisis de Detroit, 8000000.

En resumen, la producción local precisó de cierto nivel de protección estatal para su desarrollo. Una de las formas fue la política arancelaria, que evolucionó a medida que el sector se ampliaba.

\section{LA ESTRUCTURA ARANCELARIA DE LA AUTOMOTRIZ ARGENTINA (1965-1983)}

\section{El lugar de las importaciones}

Para realizar un análisis de los aranceles es necesario antes hacer una revisión de las importaciones, pues medir su evolución permitirá caracterizar la función de la estructura arancelaria.

El complejo automotriz tuvo un peso significativo en las compras del país durante la década de 1960. Luego, su participación relativa disminuyó (véase gráfica 2), esto debido a que cayó la importación propia de la actividad y al aumento de las totales de la economía.

A pesar de la caída del peso relativo no se redujo el déficit en la balanza comercial (véase gráfica 3), por lo que durante todos los años el saldo comercial fue negativo.

La balanza es negativa por el déficit en autopartes, ya que lo que se exportaba no alcanzaba a cubrir lo que se compraba: los vehículos terminados tuvieron un superávit entre 1972 y 1978, pero no fue suficiente para revertir el rojo. La actividad aún dependía de ciertos insumos externos y no generaba divisas para sostenerse, sino que las demandaba. Los aranceles y el régimen automotriz tuvieron como uno de sus objetivos reducir esta dificultad.

Como señalaron otros autores (Nofal, 1989), cabe resalta que la voluntad de las terminales para exportar dependía de las estrategias que tomaban en el centro las multinacionales, además es cierto que llegado a cierto nivel de desarrollo y capacidad instalada los propios industriales, de ambos sectores de la trama, pusieron en sus respectivas agendas la necesidad de encontrar mercados externos sostenidos para incrementar la operatoria de las firmas (Bil, Harari y Sanz, 2018). 


\section{GRÁFICA 2. PESO DE LAS IMPORTACIONES AUTOMOTRICES SOBRE LAS IMPORTACIONES TOTALES EN ARGENTINA, 1960-1983}

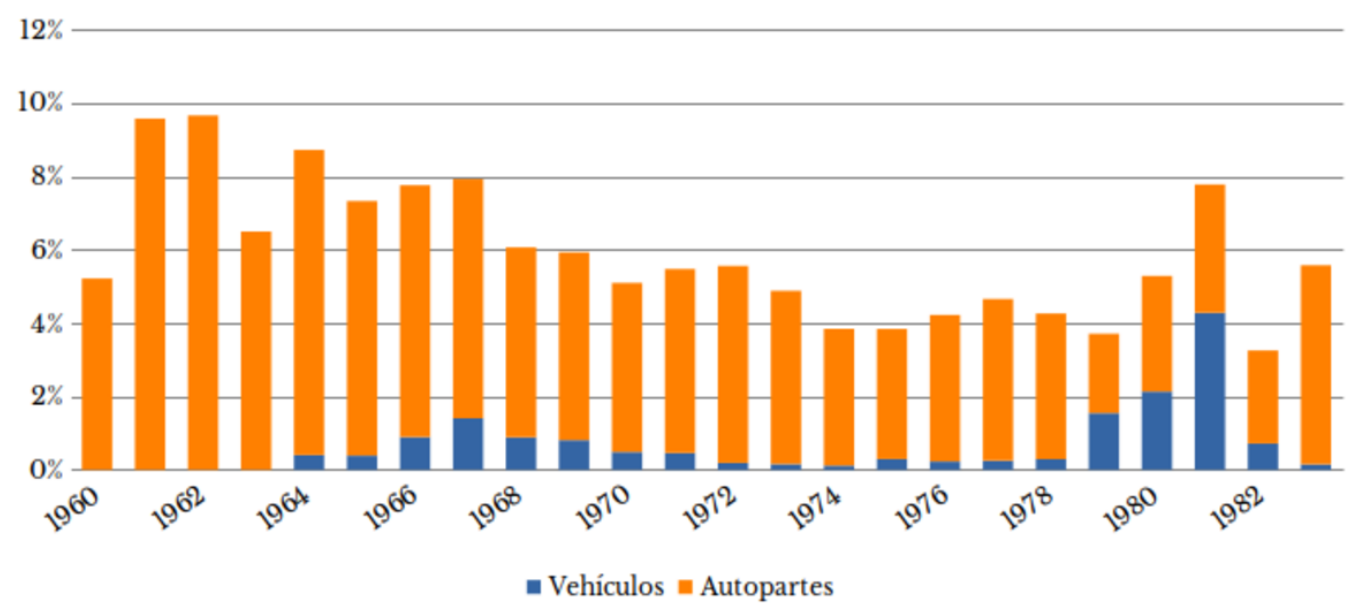
(2010).

Fuente: elaboración propia con base en Dirección Nacional de Estadísticas y Censos (1966-1983) y Ferreres

\section{GRÁFICA 3. EXPORTACIONES E IMPORTACIONES DE AUTOPARTES Y VEHÍCULOS Y SALDO DE LA BALANZA, ARGENTINA, 1966-1983 (MILLONES DE DÓLARES)}

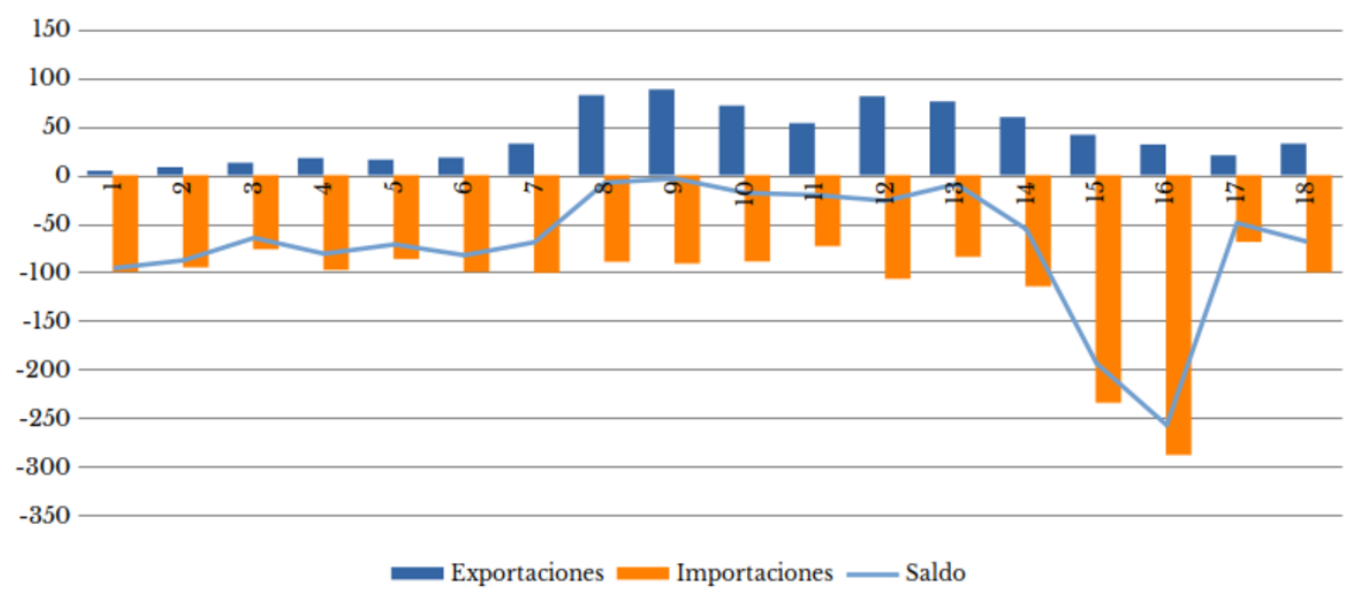

Fuente: elaboración propia con base en Dirección Nacional de Estadísticas y Censos (1966-1983).

La estructura de aranceles

El periodo analizado concentra a nuestro entender dos grandes etapas del desarrollo de la automotriz argentina: el momento de aparente consolidación, con el establecimiento de grandes participantes (1965-1977), y el momento de crisis y retiro de empresas bajo el gobierno militar (1978-1983). El análisis de la protección puede echar luz sobre las condiciones de la rama en estas etapas y su particular trayectoria. 
Existieron variadas herramientas de protección industrial, por ejemplo las normativas específicas sobre productos. Por caso, en 1965 se dispuso reducir el arancel de piezas de países de la Asociación Latinoamericana de Libre Comercio para incentivar el comercio preferencial (Boletín de la Bolsa de Comercio de Buenos Aires, 22 de febrero de 1965, p. 975); otra fue el permiso para importar máquinas, herramientas o créditos con financiación externa para inversión productiva.

En cuanto a autopartes, el régimen de 1959 estableció un nivel de integración progresivo que se calculaba sobre el valor cif del vehículo. Permitía importar con un arancel preferencial de $40 \%$ para vehículos y $20 \%$ para camiones, hasta alcanzar un 35-45\%. Se permitía importar sobre esos topes hasta un máximo del valor del $60 \%$, con $200 \%$ de arancel. Si se utilizaban plenamente estas cuotas, un fabricante podía importar hasta el $66 \%$ del valor cIF con un arancel de $62 \%$ (Fitzsimons, 2014), al tiempo que la importación de vehículos se prohíbe, por eso se sostuvieron las importaciones de piezas. Pero en 1961 se estipuló que en cuatro años se debía reducir el contenido importado a un máximo de $10 \%$. También se establecieron montos mínimos de inversión para considerar a la empresa en la promoción. Por ello, para el periodo de 1964-1966 empresas que aprovechaban la posibilidad de importar y solo ensamblar el vehículo salieron de la actividad: Cisitalia, Los Cedros, Metalmecánica, Isard, Goliath Hansa, y otras; esto provocó una primera depuración.

En 1965 se estableció otra reducción: se puso un tope máximo de integración con valor importado de $12 \%$ para camiones y en 1968 de $4 \%$ para automóviles. Para 1969, se permitió $5 \%$ con $40 \%$ de recargo. En 1971 se sancionó el Régimen de Reconversión Automotriz, analizado en la sección anterior, incluyendo al autopartismo (Sourrouille, 1980, pp. 65-66). Este nivel de integración, aunque elevado, no fue específico de Argentina, por ejemplo, Brasil, que contó con una legislación similar con una integración de 95 a 98 \% (Faria, 1970, p. 123). En Francia se integraba $100 \%$ con contenido nacional y en España con 90 a $94 \%$. En la India, con 85 \% (Baranson, 1971, pp. 27, 39). Otros contaron con menor integración: México no superó el 65 \%; Chile, 50 \% en 1966; Venezuela, $45 \%$ en 1976; Perú, $30 \%$ en 1968 y Colombia y Uruguay, $25 \%$ recién en 1973 (Lifschitz, 1982, pp. 143-145). La performance fue disímil, los mínimos de integración tenían incidencia según el país y su desarrollo previo.

En Argentina, el esquema sufrió modificaciones durante la década de 1970. En 1978 se produjo la reforma que redujo los aranceles de forma progresiva. En un contexto de crisis económica, esto dejó en evidencia los déficits de la producción local. Se dio la salida de grandes firmas como General Motors en 1978, Citröen y Chrysler en 1979, mientras que en 1980 Peugeot y Fiat vendieron su operatoria a Sevel. Eso explica en gran medida el aumento en la importación de vehículos entre 1980 y 1981.

El tipo de cambio incide también en la protección efectiva. Si hay sobrevaluación se expandirá la capacidad de compra disminuyendo el efecto del arancel nominal. Por el contrario, una moneda subvaluada adicionará una alícuota de protección. Por eso se analiza el efecto combinado, posteriormente se estudia la evolución de las importaciones del complejo con base en Dirección Nacional de Estadísticas y Censos (1966-1983), los resultados se aprecian en las gráficas 4 y 5.

Esta información permite pensar algunos problemas. En primer lugar, posibilita analizar la relación entre nivel de protección e importaciones. No observamos en esta aproximación una relación directa clara. Si bien coinciden años de descenso de protección con aumento de importaciones, hay momentos donde no se cumple esto, con niveles de protección efectiva incluso negativos (1973-1974) se importa menos que en años de protección positiva. Con protección negativa, en 
1978 y 1979 la importación es menor que el promedio de las décadas de 1960 y 1970, pero al año siguiente se disparan. A futuro es necesario incorporar otras variables, como el nivel de divisas disponibles y el peso de la producción local.

En segundo lugar, la protección del complejo era elevada. Terminales y autopartistas requirieron continua protección para operar, según los propios actores del complejo debido a sus mayores costos y menor escala en comparación con los líderes internacionales. Este punto remite al diferencial en la protección a vehículos y autopartes (véase gráfica 6).

La protección para las terminales superó a la de las autopartistas. A comienzos de la década de 1960, cuando se iniciaba la producción integral, la protección para terminales fue muy elevada: entre aranceles y valuación de la moneda la protección efectiva superaba el $700 \%$, o directamente se efectuaba la prohibición de importar. Con la posibilidad de importar hasta $60 \%$ del valor del vehículo a aranceles moderados y a la existencia de demanda insatisfecha, brindó un campo para la instalación de firmas y la generación de ganancias con base en altos precios (Sourrouille, 1980; Fitzsimons, 2014). En la etapa siguiente (1966-1983) la brecha se achicó, siendo el promedio $12 \%$; aunque a partir de la reforma de 1978 se volvió a incrementar.

¿Qué indica esta evolución? Como se observa con fuentes primarias, las terminales tenían reservas en relación con el elevado nivel de integración que demandaban los sucesivos regímenes. Incluso, firmas como Siam o Industrias Kaiser Argentina declaraban "sacrificar utilidades" para cumplir con esta normativa (Siam Di Tella Automotores, 1965, p. 199). Se considera que la situación debe entenderse en el marco de la política de compre argentino (en una perspectiva más general de ahorro de divisas que manejaron las sucesivas administraciones hasta 1976), así los aranceles permitieron un mercado protegido para las terminales y también para la industria auxiliar. La brecha entre las alícuotas para ambas actividades denunciaba una tensión permanente: permitía a las terminales vender en el mercado doméstico y compensaba en parte lo que resignaban por la política de asegurar un mercado para las autopartistas mediante el compre argentino (incluidas inversiones para integración que, como vimos, los propios empresarios denunciaban como antieconómicas). Esta situación se resolvió en parte con las nuevas reglamentaciones de fines de los setenta, en un contexto político más favorable a las terminales, momento en el cuál imponen más reivindicaciones sobre las autopartistas (Bil, 2018; Bil, Harari y Sanz, 2018). 

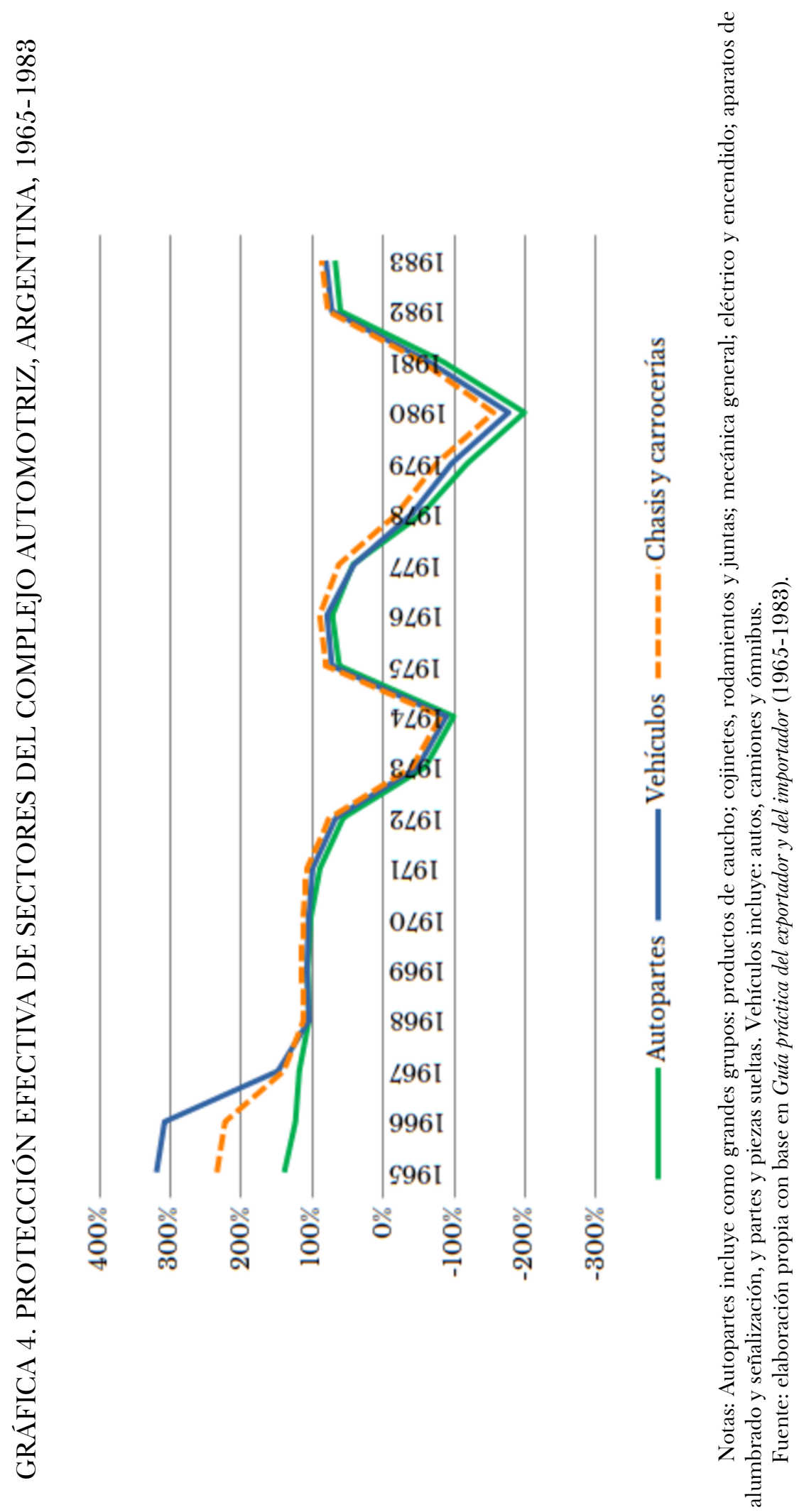


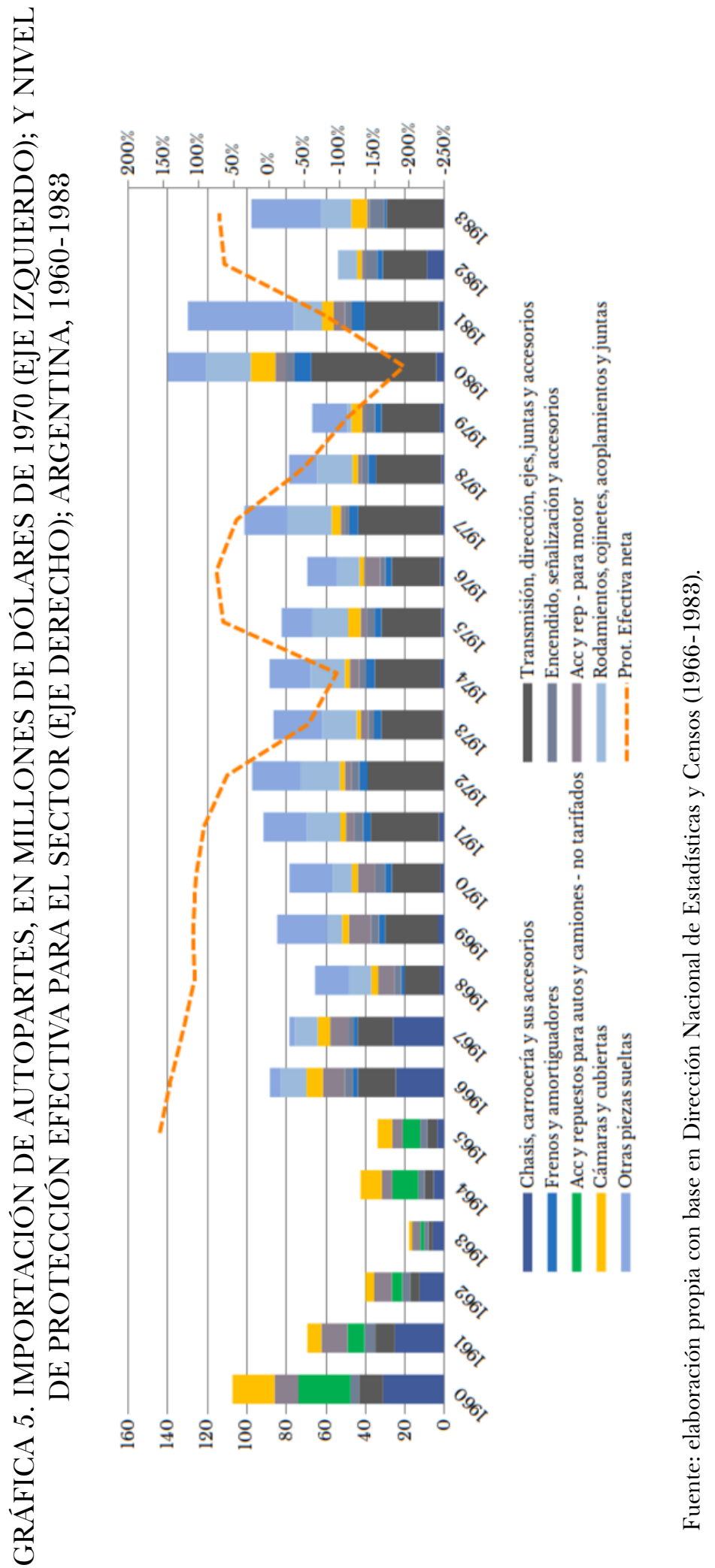




\section{GRÁFICA 6. DIFERENCIA ENTRE LA PROTECGIÓN EFECTIVA A VEHÍCULOS Y A AUTOPARTES, ARGENTINA, 1961-1983 (PROMEDIO DE LAS PARTIDAS)}

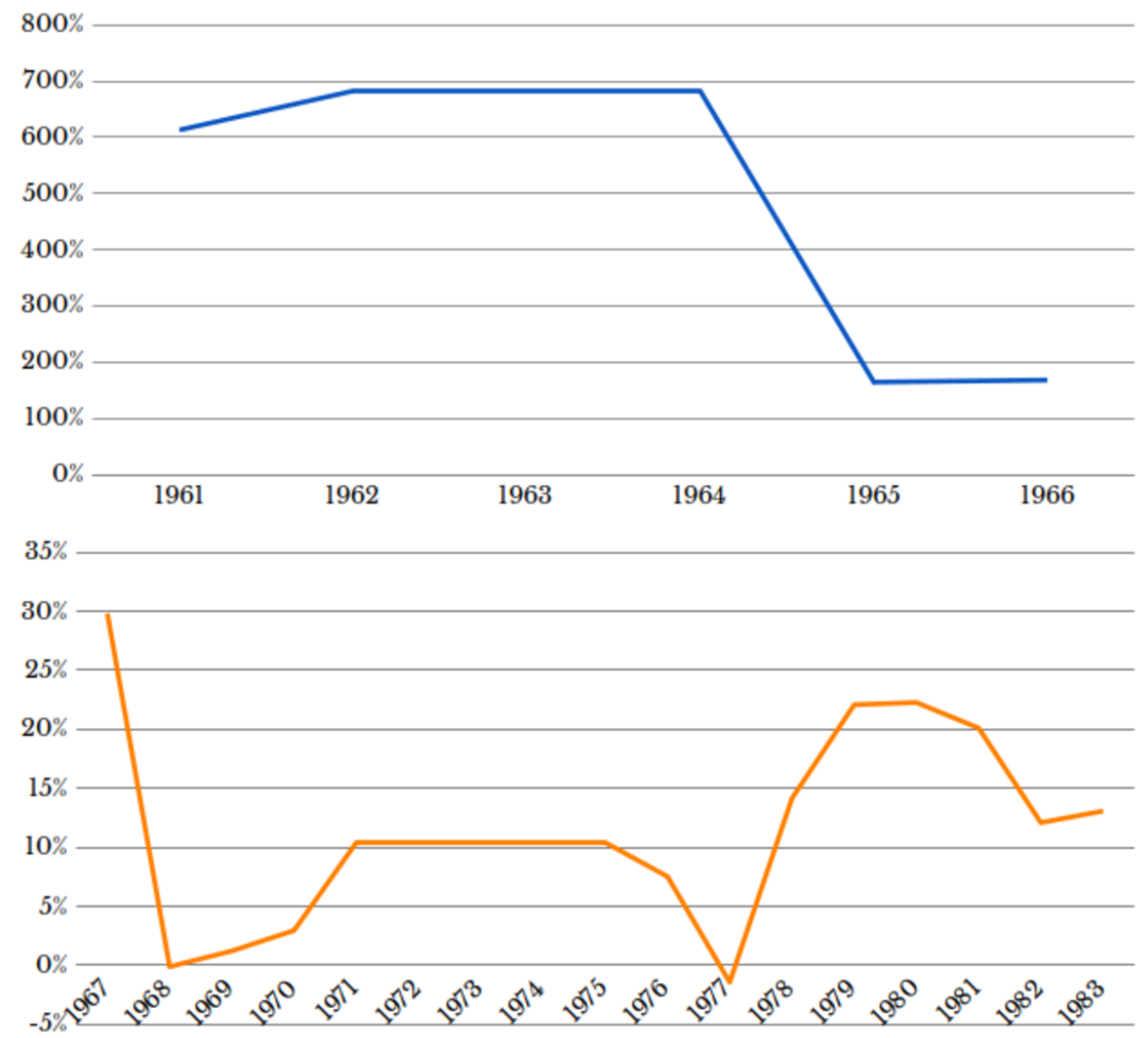

Nota: Se separó en dos gráficos porque la diferencia entre 1961 y 1966 es demasiado grande. En el periodo de 1963 a 1964 la brecha entre la protección de terminales y autopartes es infinita, debido a que la importación de vehículos fue prohibida. Se optó por repetir la brecha de 1962 para completar la gráfica.

Fuente: elaboración propia con base en Dirección Nacional de Estadísticas y Censos (1966-1983).

\section{Conclusiones}

Se analizó la protección arancelaria del complejo automotriz argentino en el periodo de 19651983. Además se reconstruyó la protección efectiva (combinación de aranceles y efecto del tipo de cambio) y sus consecuencias en la balanza comercial con el objetivo de analizar el papel de la política estatal en la acumulación de capital en la rama.

Las normativas de fomento para el sector automotriz toman forma en momentos de dificultades para importar y de consecuente envejecimiento del parque. En este contexto, ante la escasez de divisas (problema recurrente en la historia argentina desde mediados del siglo $\mathrm{xx}$ ), se profundizaron medidas de protección en general, y en particular para el sector automotriz. Se edificó una protección para el complejo frente a la menor competitividad de esta industria naciente en el contexto internacional. Al calor de estas medidas, creció la industria autopartista con expansión de firmas 
nacionales e instalación o licencias de extranjeras, y se instalaron firmas multinacionales en la etapa terminal, donde se estimulaba la radicación. Como estudiaron varios especialistas, este esquema sufriría modificaciones hasta su relativa liberalización a partir del golpe militar de 1976.

Encontramos que la estructura, que tenía como uno de sus objetos el ahorro de divisas, impactó de manera peculiar en la configuración del complejo y tuvo distintas recepciones en los dos sectores fundamentales. Esta situación llevó a indagar en la relación entre terminales y autopartistas, por lo que se reconstruyó entonces el nivel de protección a partir de los límites de la acumulación de capital en este sector.

Se halló, a partir de los datos empíricos, que a pesar de los regímenes la balanza continuó siendo deficitaria. En parte porque se mantuvieron niveles de importación de partes y piezas, y en parte al hecho de que la producción local no logró hacer pie en mercados externos.

Terminales y autopartistas reconocían problemas en la actividad, aunque deslindaban responsabilidades. Las primeras no acordaban con el nivel de integración exigido y reclamaban flexibilizar el régimen, mientras las autopartes solicitaban más protección. Esta tensión afloró en las negociaciones periódicas de los regímenes. Como resultado, las terminales impusieron el grueso de sus reivindicaciones recién bajo el gobierno militar.

$\mathrm{Al}$ revisar la protección efectiva, se encontró que al inicio fue más elevada, posteriormente, progresivamente, se redujo. Esto podría relacionarse con la mejora competitiva (incorporación de tecnología, racionalización) una vez instalado el complejo y depurado de las empresas menos eficientes. Existió una diferencia entre el nivel de protección de ambas etapas. Durante la década de 1960 la brecha fue muy elevada, reduciendo a un 10 \% promedio a finales de esa década. Volvió a aumentar luego de 1978. Se considera que el fenómeno se relaciona con la política de protección general al complejo. Ante la imposibilidad de las terminales para importar más piezas, se les subsana con un elevado arancel que les permitiría compensar en el mercado interno lo que resignan al tener que abastecerse en el mercado local.

En la investigación no se encontró una relación concluyente entre las variables protección e importación. Por el momento no es posible concluir cuál es el efecto concreto de las normativas sobre las compras al extranjero. Para ello, resta analizar otros indicadores del comercio exterior, como los acuerdos con otros países de la Asociación Latinoamericana de Libre Comercio, permisos a las terminales para comprar piezas especiales, entre otras.

En definitiva, se espera que este trabajo sirva como base para profundizar los ejes aquí planteados, y también para utilizar como insumo para otras indagaciones, como el estudio de la estructura de costos de la actividad.

\section{LISTA DE REFERENCIAS}

Altimir, O., Santamaría, H. y Sourrouille, J. (1967). Los instrumentos de promoción industrial en la postguerra. Desarrollo económico, 7(27), 149-172.

Asociación de Fabricantes de Automotores. (1969). Los factores que distorsionan la formación de los costos en la industria automotriz. Buenos Aires: Autor.

Balassa, B. (1975). Latin American Trade Policies in the 1970's: A Comment. The Quarterly Journal of Economics, 89(3), 483-486. DOI: 10.2307/1885268

Baranson, J. (1971). La industria automotriz en los paises en desarrollo. Madrid: Tecnos. 
Barbero, M. I. y Motta, M. S. (2007). Trayectoria de la industria automotriz en la Argentina desde sus inicios hasta fines de la década de 1990. En M. Delfini (ed.), Innovación y empleo en tramas productivas de Argentina. Buenos Aires: Prometeo.

Belini, C. y Rougier, M. (2008). El Estado empresario en la industria argentina: Conformación y crisis. Buenos Aires: Manantial.

Berlinski, J. (1977). Protección arancelaria de actividades seleccionadas de la industria manufacturera argentina. Buenos Aires: Mimeo.

Berlinski, J. (1978). La estructura de protección de actividades seleccionadas (evidencias y reflexiones sobre el caso argentino). Presentado en XIII Reunión Anual de la Asociación Argentina de Economía Política, Buenos Aires, Río Tercero.

Berlinski, J. y Schydlowsky, D. (1982). Argentina. En B. Balassa (ed.), Development strategies in semi-industrial economies. Baltimore: Johns Hopkins University Press.

Bil, D. (2017). La trayectoria de tres compañías autopartistas en Argentina en el periodo de formación del sector automotriz (1951-1967). H-Industria, 11(20), 43-63.

Bil, D. (2018). Los industriales automotores y la crisis de la década de 1980 en Argentina. Economía del Caribe, 21, 105-130.

Bil, D. y Bastida, J. (2014). Midiendo la rentabilidad de la industria argentina. Un ejercicio con las ramas autopartista y automotriz durante el periodo de sustitución (1948-1967). Ciclos en la Historia, la economía y la sociedad, 23(44).

Bil, D., Dachevsky, F. y Kornblihtt, J. (2010). Debates sobre el desarrollo industrial en Argentina (1930-1976). En E. Sartelli (ed.), La crisis orgánica de la sociedad argentina. Buenos Aires: Editorial de la Facultad de Filosofía y Letras, Universidad de Buenos Aires.

Bil, D., Harari, I. y Sanz, G. (2018). ¿Liberales o proteccionistas? Estrategias económicas contrapuestas en la cadena automotriz argentina en las décadas del 60 y 70 . Avances del CESOR, 15(18), 1-21.

Boletín de la Bolsa de Comercio de Buenos Aires (1965, febrero 22). 975.

Broner, J. y Larriqueta, D. (1969). La revolución industrial argentina. Buenos Aires: Sudamericana.

Cámara Industrial de Fabricantes de Autopartes de la República Argentina [CIFARA]. (1970). Estudio técnico-económico de la industria nacional del transporte. Buenos Aires: Autor.

Carrera, J. I. (2007). La formación económica de la sociedad argentina. Buenos Aires: Imago Mundi.

Castellani, A. (2009). Estado, empresas y empresarios: La construcción de ámbitos privilegiados de acumulación entre 1966 y 1989. Buenos Aires: Prometeo Libros.

Cimillo, E., Lifschitz, E., Gastiazoro, E., Ciafardini, H. y Turkieh, M. (1973). Acumulación y centralización del capital en la industria argentina. Buenos Aires: Tiempo Contemporáneo.

Dadone, A. y Swoboda, C. (1979). La reforma arancelaria argentina. Asociación Argentina de Economía Política, 14, 411-440.

Dirección Nacional de Estadísticas y Censos (1966). Anuario de Comercio Exterior Argentino. Argentina: Autor.

Fajnzylber, F. (1983). La industrialización trunca de América Latina. México: Centro de Economía Transnacional/Editorial Nueva Imagen.

Faria, A. (1970). A indústria automotriz no Brasil. Parte B: indústria de partes e peças. Santiago (Documento Informativo, No 9). Santiago: CEPAL.

Ferreres, O. J. (2010). Dos siglos de economía argentina: 1810-2004 historia argentina en cifras. Buenos Aires: Norte y Sur. Recuperado de http://biblio.econ.uba.ar/cgi-bin/koha/opac-detail.pl? biblionumber $=146592$ 
Fiszbein, M. y Rougier, M. (2013). Crecimiento desbalanceado y estructura productiva desequilibrada en Argentina (1945-1976): Problemas e ideas del modelo industrial. En Estudios sobre la industria argentina (pp. 49-74). Buenos Aires: Lenguaje Claro.

Fitzsimons, A. (2013). ¿Por qué las multinacionales automotrices instalan fábricas en América Latina? Desarrollo del proceso de trabajo y organización internacional de las empresas de la rama (1910-1970). Presentado en VIII Jornadas de Estudios Sociales de la Economía, Buenos Aires.

Fitzsimons, A. (2014). Estado y acumulación de capital en Argentina: La expansión de las empresas extranjeras entre 1958 y 1963 (Tesis de doctorado). Universidad de Buenos Aires, Argentina.

Fitzsimons, A. (2016). Procesos de trabajo e internacionalización del capital: determinantes globales del "atraso" tecnológico de la industria automotriz argentina en las décadas de $1950 \mathrm{y}$ 1960. Trabajo y Sociedad, 26, 225-240.

Fitzsimons, A. y Guevara, S. (2018). La industria automotriz argentina y sus fuentes de ganancia: Un análisis de largo plazo (1960-2013). América Latina en la Historia Económica, 25(1), 239274. doi: 10.18232/alhe.859

Gereffi, G. (1999). International Trade and Industrial Up-Grading in the Apparel Commodity Chain. Journal of International Economics, 48 (1), 37-40. Doi: 10.1016/S0022-1996(98)000750

Grinberg, N. (2011). Transformations in the Korean and Brazilian Processes of Capitalist Development Between the Mid-1950s and the Mid-2000s the Political Economy of Late Industrialization (Tesis de doctorado). London School of Economics and Political Science, Londres.

Guía Práctica del Exportador y del Importador (1962). 6(68).

Guía Práctica del Exportador y del Importador (1964). 8(85).

Guía práctica del exportador y del importador $(1965,1983)$.

Harari, I. (2010). ¿Vehículos del Imperialismo? La industria automotriz y las deficiencias de la teoría del imperialismo/dependentismo. Razón y Revolución, 20, 29-47.

Harari, I. (2011). Evolución y transformación del proceso de trabajo en la industria automotriz entre 1952 y 1976 (Tesis de doctorado). Universidad de Buenos Aires, Buenos Aires.

Industrias Kaiser Argentina (1963). Memoria y Balance del $8^{\circ}$ Ejercicio finalizado el 30 de junio de 1962. Boletín de la Bolsa de Comercio de Buenos Aires.

Katz, J. y Kosacoff, B. (1989). El proceso de industrialización en Argentina: Evolución, retroceso y prospectiva. Buenos Aires: Comisión Económica para América Latina y El Caribe.

Kosacoff, B. y Azpiazu, D. (1989). La industria argentina: desarrollo y cambios estructurales. Buenos Aires: CEPAL.

Lifschitz, E. (1982). El complejo automotor en México y América Latina. México: Universidad Autónoma Metropolitana.

Mercado (1980, mayo 1). 552, 26.

Mercado (1982, enero 28). 641.

Nofal, M. B. (1989). Absentee entrepreneurship and the dynamics of the motor vehicle industry in Argentina. Nueva York: Praeger.

Nogues, J. J. (1982). Política arancelaria y de promoción de exportaciones: elementos para su formulación. Económica, 28(3), 211-243.

Parrellada, E. (1970). La industria automotriz en la Argentina. Santiago: Comisión Económica para América Latina y El Caribe.

Pinazo, G. D. (2015). El desarrollismo argentino: una mirada crítica desde la industria automotriz. Argentina: Universidad Nacional de General Sarmiento. 
Racanello, M. (2010). Del Segundo Plan Quinquenal a la Convertibilidad: La industria del tractor frente a los cambios estructurales de la economía. En M. Rougier (ed.), Estudios sobre la industria argentina: políticas de promoción y estrategias empresariales-2. Buenos Aires: Lenguaje Claro.

Remes, J. (1958). Algunos resultados de la política desarrollista (1958-1964): El caso de la industria automotriz. En Problemas económicos argentinos. Diagnóstico y política. Buenos Aires: Macchi.

Rezk, E. y Ruatta, O. B. (1980). Algunas consideraciones sobre el efecto protección en la estructura arancelaria argentina. Córdoba: Facultad de Ciencias Económicas.

Rossi, J. (1960). Estado actual y perspectivas de la industria automotriz. Boletín de la Bolsa de Comercio de Buenos Aires, 56(2882).

Rougier, M. (2012). La economía del peronismo: una perspectiva histórica. Buenos Aires: Editorial Sudamericana.

Rougier, M. y Odisio, J. (Eds.). (2016). Estudios sobre planificación y desarrollo. Buenos Aires: Lenguaje Claro Editora.

Schteingart, D., Rougier, M. y Odisio, J. (eds.). (2016). La restricción externa en el largo plazo: Argentina, 1960-2013. En Estudios sobre planificación y desarrollo (pp. 325-365). Buenos Aires: Lenguaje Claro Editora.

Siam Di Tella Automotores. (1965, junio 7). Memoria y Balance Siam del $6^{\circ}$ Ejercicio cerrado el 31 de diciembre de 1964. Boletín de la Bolsa de Comercio de Buenos Aires, 59(3135).

Sourrouille, J. V., Kosacoff, B. P. y Lucangeli, J. (1985). Transnacionalización y política económica en la Argentina. Buenos Aires: Comisión Económica para América Latina y El Caribe. Recuperado de https://idl-bnc-idrc.dspacedirect.org/handle/10625/8903

Sourrouille, J. V. (1980). El complejo automotor en Argentina: Transnacionales en América Latina. México: Editorial Nueva Imagen.

Szychowski, M. L. (1988). La protección efectiva (tratamiento de los insumos de bienes domésticos). Económica, 34(2), 275-297.

Taraciuk, T. (2015). Julio Broner: argentino por opción. Buenos Aires: Autor.

Teitel, S. y Thoumi, F. E. (1986). From Import Substitution to Exports: The Manufacturing Exports Experience of Argentina and Brazil. Economic Development and Cultural Change, 34(3), 455-490. DOI: 10.1086/451545

Wainer, P. (1970). La protección aduanera efectiva en la República Argentina. Presentado en Reunión Anual de la Asociación Argentina de Economía Política, Buenos Aires.

Wilkins, M. y Hill, F. E. (2011). American business abroad: Ford on six continents. Nueva York: Cambridge University Press. 